\title{
REPRESENTACIONES SOCIALES (RS) DEL CUIDADO DE ENFERMERÍA DESDE UN ENFOQUE HUMANÍSTICO. HOSPITAL ESTATAL CERRO DE PASCO, PERÚ
}

\author{
Social representations (SR) of nursing care from a humanistic approach at the State Hospital \\ Cerro de Pasco, Perú
}

Nelly Marleni Hinostroza Robles ${ }^{1 a, b, c}$, Martha Nicolasa Vera Mendoza ${ }^{2 a, b, c}$

${ }^{1}$ Profesor Principal, Escuela de Formación Profesional de Enfermería, Facultad de Ciencias de la Salud, Universidad Nacional Daniel Alcides Carrión, Cerro de Pasco, Perú, ${ }^{2}$ Profesor Principal, Departamento Académico de Enfermería, Facultad de Medicina de la Universidad Nacional Mayor de San Marcos, Lima, Perú.

${ }^{a}$ Lic. En Enfermería. ${ }^{b}$ Maestro en Enfermería, ${ }^{c}$ Doctor en Ciencias de la Salud

\section{RESUMEN}

El cuidado humanístico es el referente de la práctica de enfermería, guiada por su filosofía explicita que confiere identidad al acto científico, considerando la interacción transpersonal enfermera-paciente y familiar, en la práctica clínica y comunitaria. Objetivo: Describir y construir las representaciones sociales (RS) del cuidado de enfermería desde un enfoque humanístico. Material y Métodos: Estudio cualitativo-descriptivo interpretativo realizado en el Hospital Estatal de Cerro de Pasco Perú, participando veintiún enfermeras (os) de los servicios de hospitalización. Se utilizó como técnicas e instrumentos de recolección de datos; la observación participante y la entrevista en profundidad. Resultados: Se muestra la emergencia de cuatro categorías: 1. Identificando el significado de ser enfermera (o) 2. Identificando conocimientos del significado de cuidado humanístico según la enfermera(0) 3. Fortalezas y debilidades en la interacción profesional 4. Actitudes y valores relacionados al cuidado humanístico en el entorno de los servicios hospitalarios. Conclusiones: El cuidado de enfermería en los servicios hospitalarios representado desde la matriz socio estructural de la enfermera (o) como profesional de las ciencias de la salud, evidencian que existen debilidades en la interacción transpersonal propio del cuidado humanístico por “....realidad de difícil afrontamiento en el hospital estatal, que genera sobrecarga laboral y estrés en el profesional, e insatisfacción de pacientes y familiares, alterando los contenidos afectivos y conativos del cuidado", que demuestran la separación entre valores científicos y valores sociales.

Palabras Clave: Cuidado de enfermería, humanismo, representaciones sociales.

\section{SUMMARY}

Humanistic care is the referent of nursing practice, guided by its explicit philosophy that confers identity to the scientific act, considering the transpersonal nurse-patient and family interaction, in clinical and community practice. Objective: To describe and construct social representations (SR) of nursing care from a humanistic perspective. Material and methods: QualitativeDescriptive Interpretive in State Hospital, Cerro de Pasco Perú, with twenty-one nurses of hospitalization services. It was used as techniques and instruments of data collection; participant observation and in-depth interview. Results: The emergence of four categories is shown 1. Identifying the meaning of being a nurse 2. Identifying knowledge of the meaning of humanistic care according to the nurse 3. Strengths and weaknesses in professional interaction 4 . Attitudes and values related to humanistic care in the hospital services environment. Conclusions: Nursing care in hospital services represented by the socio-structural matrix of the nurse (or) as a professional in the health sciences, evidences that there are weaknesses in the transpersonal interaction characteristic of humanistic care for "... a reality of difficult coping in the state hospital, which generates work overload and professional stress, and patient and family dissatisfaction, altering the emotional and conative contents of care, "which demonstrate the separation between scientific values and social values.

Keywords: Nursing care, humanism, social representations. 


\section{INTRODUCCIÓN.}

El cuidado humanístico constituye el fundamento humano y singular de la enfermería, que la diferencia de otras disciplinas del área de salud, está centrado en la interacción transpersonal enfatizando los contenidos afectivos y conativos entre enfermera-paciente, guiada por la filosofía existencial, fenomenológica y humanista en el ambiente clínico y comunitario, en el intervienen factores como cultura organizacional y coordinación para mantener la unidad de servicio durante el proceso del cuidado( $1,2,3)$.

Ante el riesgo de deshumanización en el cuidado del paciente a causa de la gran restructuración administrativa de la mayoría de los sistemas de cuidados de salud en el mundo, se hace necesario el rescate del aspecto humano, espiritual y transpersonal en la práctica clínica, administrativa, educativa e investigativa por parte de la profesional de enfermería (4).

Enfermería necesita una revolución humanística que la vuelva a centrar en su función cuidadora (2). El Colegio de Enfermeros del Perú analiza la situación de Enfermería, reflexiona que se precisa mejorar la práctica profesional reconociendo que debe ser un trabajo de transformación, que responda a los cambios de patrones de la salud relacionados con la modernización. La administración enfatiza la primacía de resultados inmediatos potencialmente deshumanizantes para pacientes y personal. Frente a esta realidad, se precisa hacer investigaciones de cuidado humanizado para el fortalecimiento de los campos de la práctica profesional (5).

Un estudio en el hospital de Huacho, Perú revela que existe una percepción muy baja del cuidado humanizado que realiza el profesional de enfermería en favor de los pacientes, ésta se basa en el hecho de que el 39\% sólo algunas veces lo perciben y el $24,7 \%$ nunca lo percibieron (6).

Las Representaciones Sociales (RS) nos permiten entender la dinámica de las interacciones sociales y aclaran los determinantes de las prácticas sociales, pues la representación, el discurso y la práctica se generan mutuamente y constituye un paso significativo para la modificación de una representación y por ende de una práctica social (7). Para el abordaje de las (RS) en la perspectiva psicosociológica se asume una posición epistemológica especifica que involucra la comprensión del sujeto social enfermera y el objeto o fenómeno social; la práctica del cuidado humanístico en un ambiente hospitalario, tan cuestionado en la actualidad. La teoría de las (RS) nos permite conocer saberes producidos en su práctica cotidiana del saber común expresados mediante su lenguaje y la matriz socio estructural en las que están inmersas las personas porque define la lectura de su realidad social (8).

El objetivo de la investigación fue describir y construir las representaciones sociales del cuidado de enfermería desde un enfoque humanístico en el Hospital Estatal de Cerro de Pasco Perú.

\section{MATERIAL Y METODOS}

El abordaje metodológico fue el cualitativodescriptivo interpretativo fundamentado en la teoría de las (RS) de Serge Moscovici y Denise Jodelet (8) a través de la objetivación y anclaje que permitió entender la dinámica de las interacciones sociales. La investigación cualitativa cumplió las siguientes características; holística porque considero la integralidad del sujeto de estudio, empírica orientado al trabajo de campo, interpretativa considerando las bases filosóficas, sociológicas, psicológicas, antropológicas y éticas del cuidado de enfermería, con empatía y compatía, considerando el contexto.

Para el acceso al trabajo de campo se realizó trámites administrativos, con Proveído Favorable de la Jefatura de Recursos Humanos y Jefatura de Enfermería, los participantes del estudio fueron informados de los aspectos éticos esenciales de su participación en la investigación, quienes brindaron el consentimiento documentado para iniciar la investigación. 
Las técnicas e instrumento utilizados fueron la observación participante, la entrevista en profundidad y una guía semiestructurada, considerando las doce tareas fundamentales propuesta por J. Spradley, (9) cumpliendo un ciclo de fases de recogida y análisis de datos en un proceso dialéctico con rigor científico.

Los discursos de los informantes fueron transcritos en cuadros comparativos en formato Word asignándole un código y denominación a cada participante de la siguiente manera: Enfermera (o) $N^{\circ} 1\left(E_{1}\right)$ hasta Enfermera $N^{\circ} 21\left(E_{21}\right)$. Para el análisis semántico de los discursos se consideró los significantes y significados en tres momentos: 1. Codificación abierta. 2. Codificación axial. 3. Codificación selectiva (8). La codificación abierta permitió identificar las oraciones o frases relacionadas al estudio que constituyeron unidades de significado y códigos vivos. La codificación axial permitió agrupar las unidades de significados y los códigos vivos, de los cuales emergieron las subcategorías y categorías, a partir de las cuales se genera la construcción de las representaciones sociales evidenciadas en los esquemas o núcleos figurativos.

\section{RESULTADOS.}

En la investigación participaron veintiún enfermeras (os) de los servicios de hospitalización de medicina, cirugía y pediatría, $90 \%$ de sexo femenino y $10 \%$ sexo masculino, cuyas edades comprendían entre 30 y 62 años, con tiempo de servicio entre 6 y 36 años como enfermeras asistenciales, el $67 \%$ son casadas, el $33 \%$ son solteras, el $71 \%$ son enfermeras especialistas, el $24 \%$ son enfermeras generales, el $5 \%$ enfermeras con grado de maestría, el $62 \%$ son nombradas y el $38 \%$ son contratadas.

El proceso de codificación permitió la emergencia de cuatro categorías, y sus respectivas subcategorías que reflejan una mayor tendencia; una tendencia intermedia y una menor tendencia de los discursos que a continuación se presentan mediante la objetivación y el anclaje.

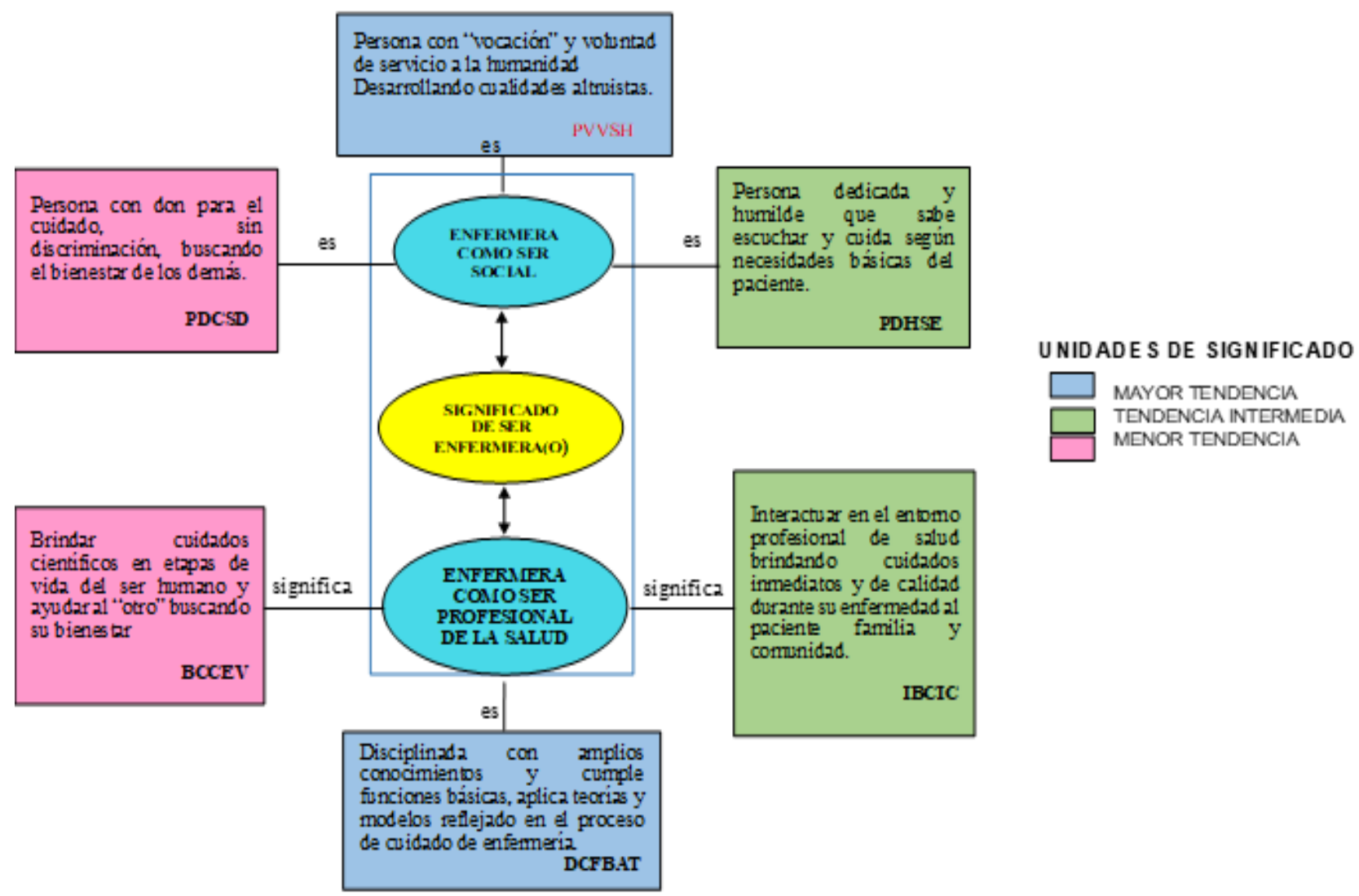

Figura 1. Identificando el significado de ser Enfermera (o) (RS) 


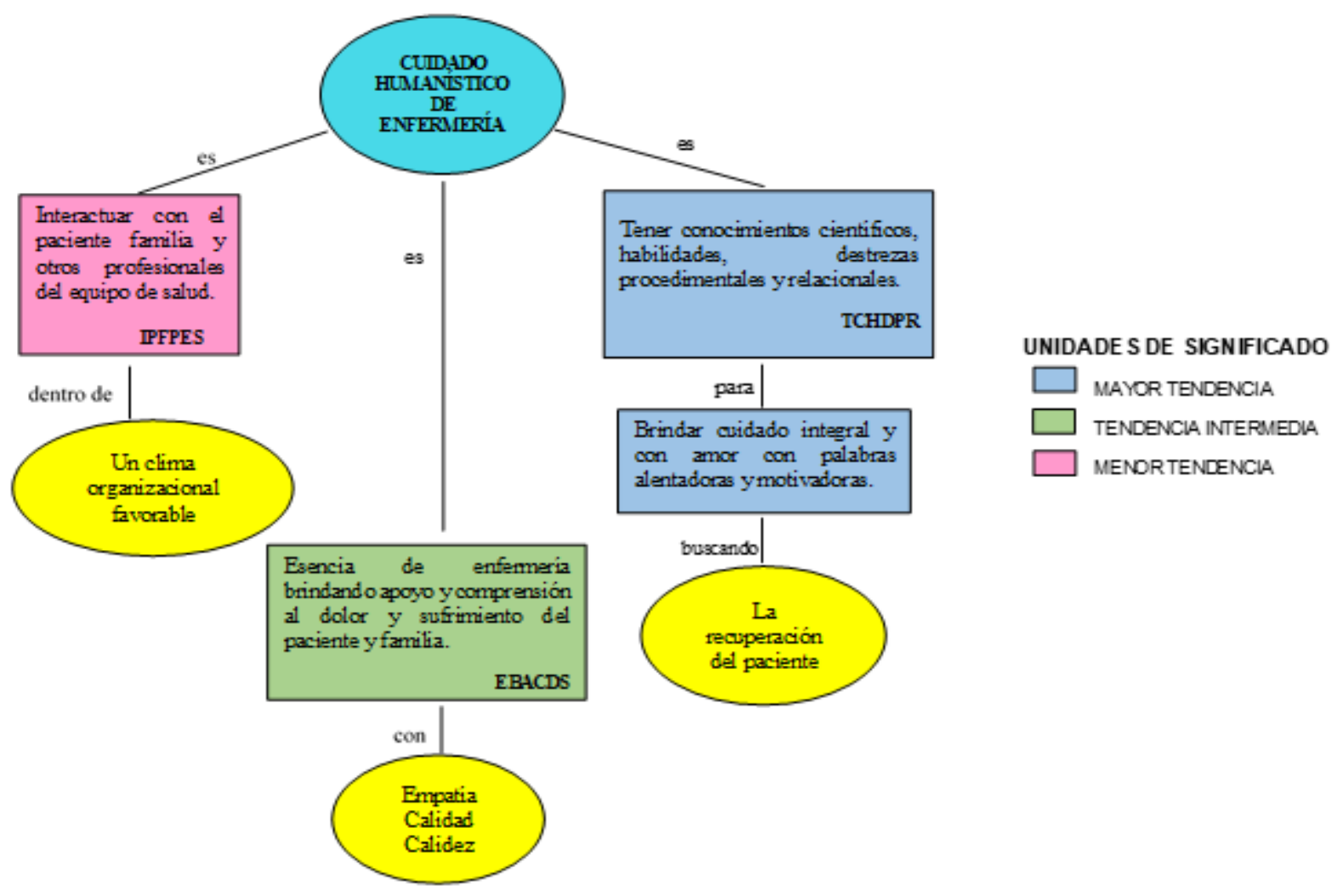

Figura 2. Identificando el significado de cuidado humanístico según la Enfermera (o) en los servicios hospitalarios (R.S.)

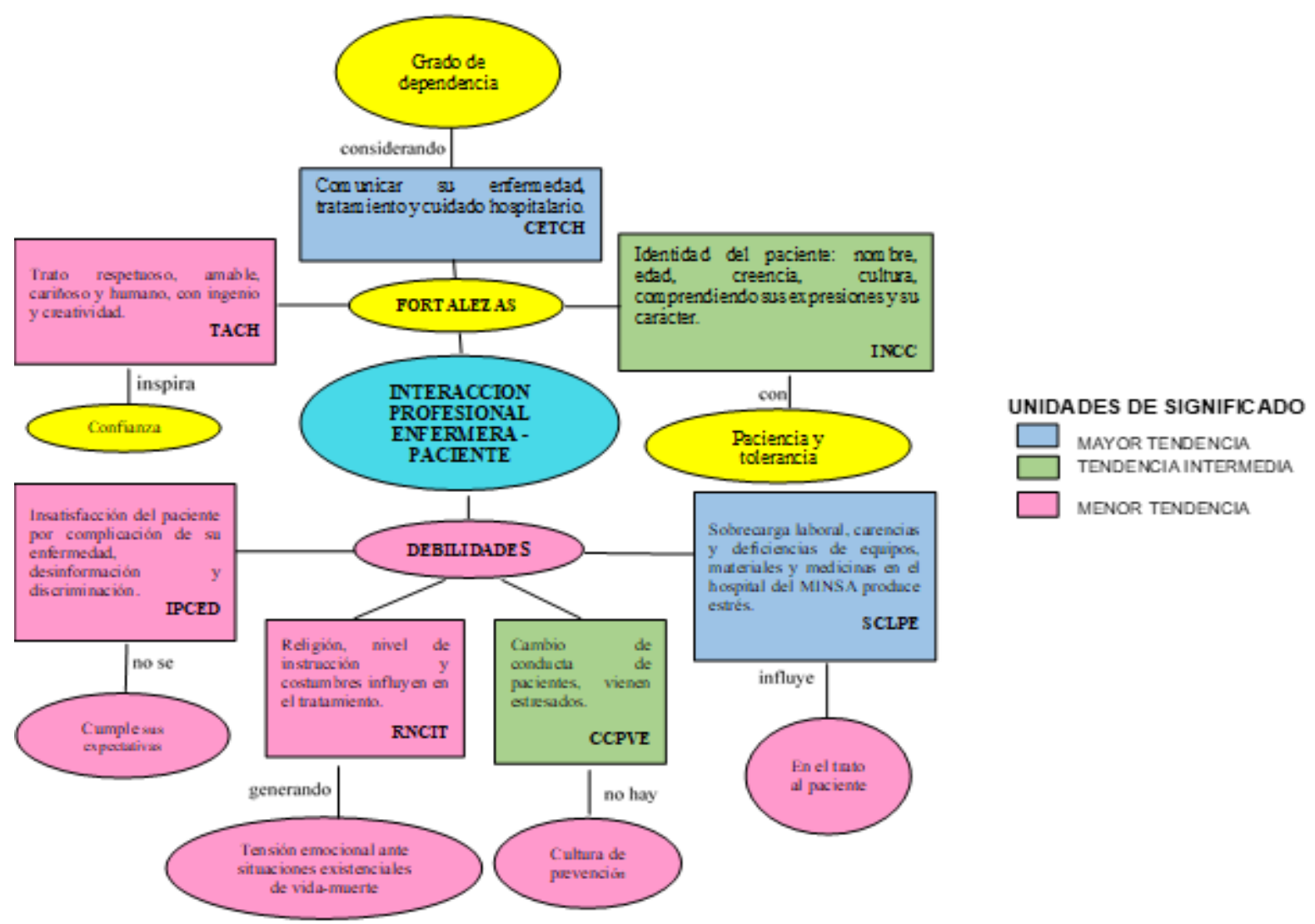

Figura 3a. Identificando la interacción profesional entre enfermera y paciente en los servicios hospitalarios (RS) 


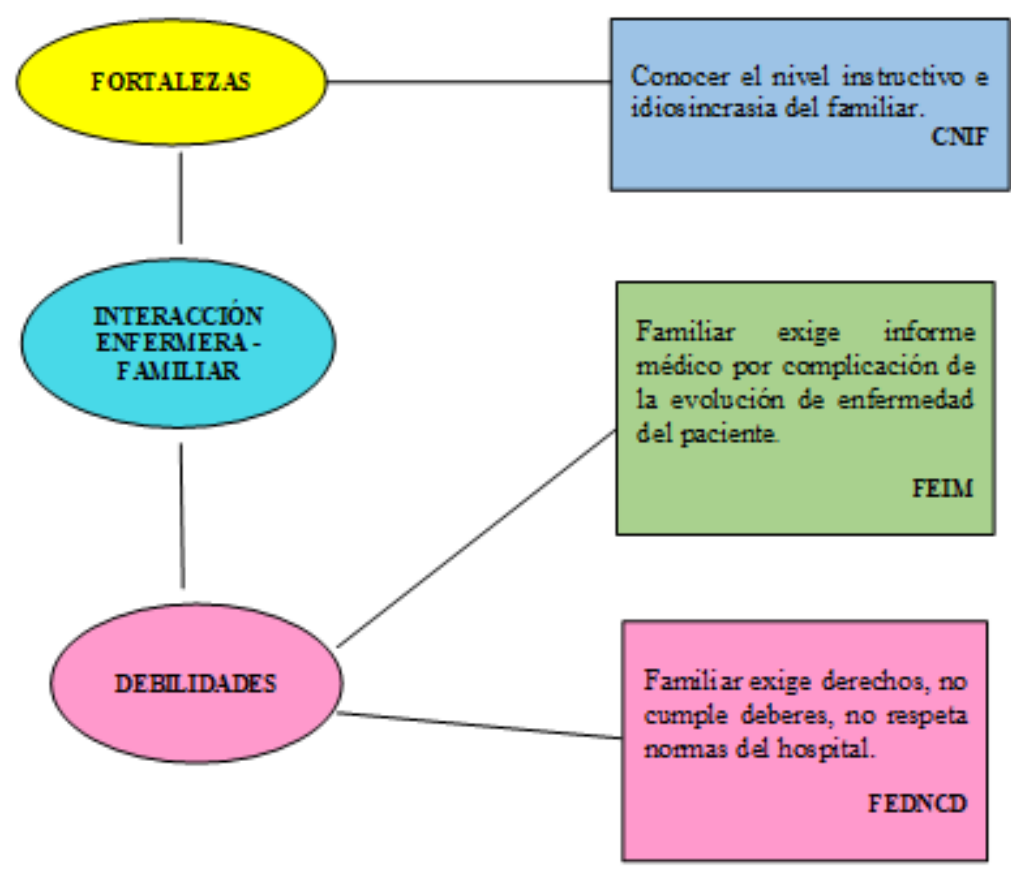

UNIDADES DE SIGNIFICADO

médico por complicación de

la evolución de enfermedad

EIM

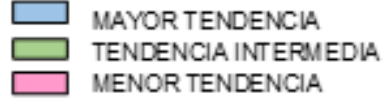

Figura 3b. Identificando la interacción profesional entre enfermera y familiar en los servicios hospitalarios (RS)

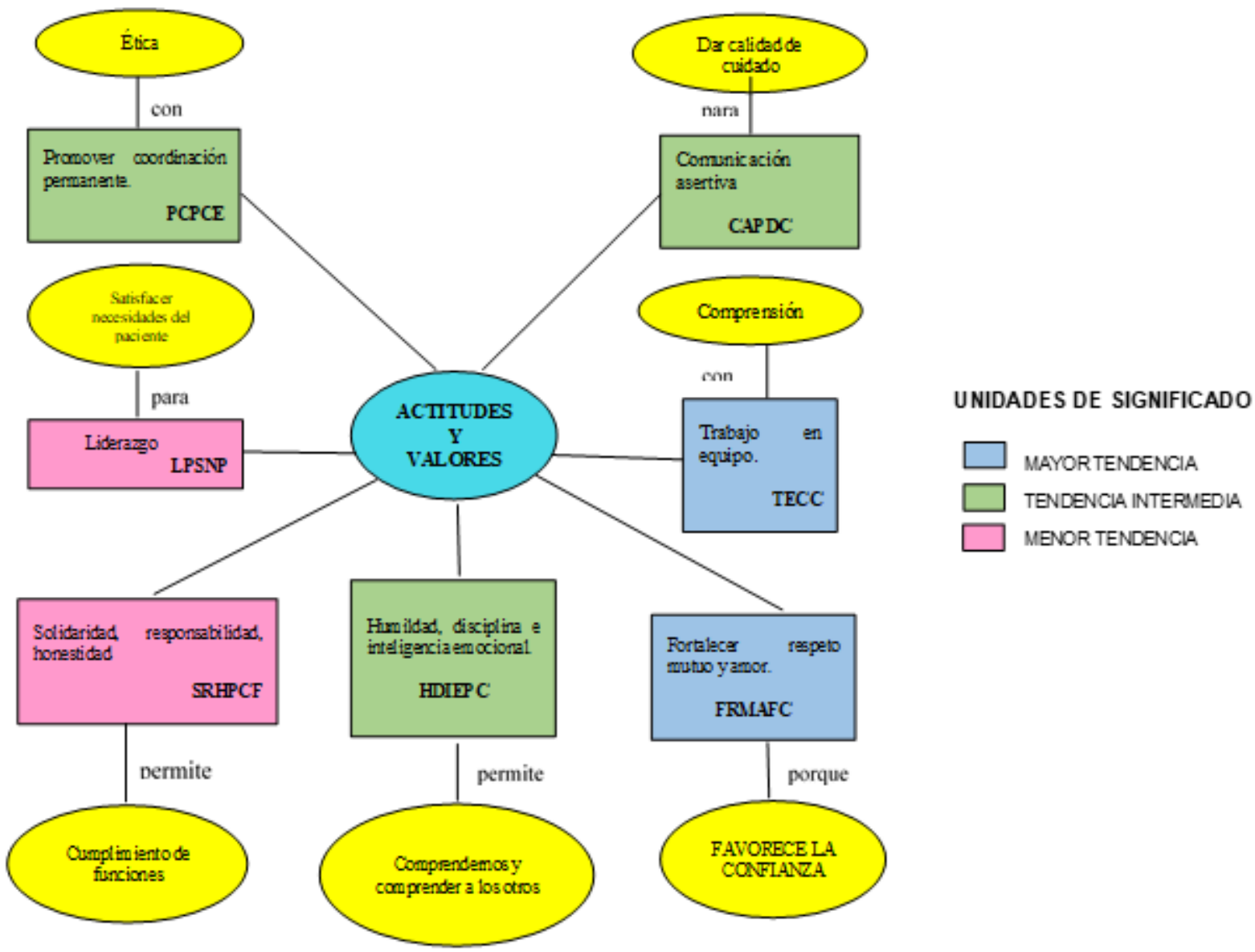

Figura 4. Actitudes y valores de la Enfermera (o) relacionados al cuidado humanístico en los servicios hospitalarios (RS) 
Tabla 1. Representaciones sociales (RS) del cuidado de enfermería

\begin{tabular}{|c|c|}
\hline CATEGORIAS & SUBCATEGORIAS \\
\hline $\begin{array}{l}\text { 1. Significado de ser } \\
\text { enfermera (o) }\end{array}$ & $\begin{array}{l}\text { - Persona con "vocación" y voluntad de servicio a la humanidad, } \\
\text { desarrollando cualidades altruistas. } \\
\text { - Profesional que cumple funciones básicas aplicando teorías y } \\
\text { modelos reflejados en el proceso de cuidado de enfermería. } \\
\text { - Brinda cuidados científicos inmediatos y de calidad en etapas de } \\
\text { vida. }\end{array}$ \\
\hline $\begin{array}{l}\text { 2. Significado de } \\
\text { cuidado humanístico } \\
\text { según la enfermera (o) }\end{array}$ & $\begin{array}{l}\text { - Tener conocimientos científicos, habilidades y destrezas } \\
\text { procedimentales y relacionales para el cuidado integral con } \\
\text { palabras alentadoras y motivadoras. } \\
\text { - Esencia de enfermería brindando apoyo y compresión en el dolor } \\
\text { y sufrimiento del paciente y familia. } \\
\text { - Es interactuar con el paciente, familia y otros profesionales de } \\
\text { equipo de salud. }\end{array}$ \\
\hline $\begin{array}{c}\text { 3. Fortalezas y } \\
\text { debilidades en la } \\
\text { interacción profesional }\end{array}$ & $\begin{array}{l}\text { - Fortalezas en la Interacción enfermera-paciente: } \\
\checkmark \quad \text { Identidad del paciente comprendiendo su carácter con } \\
\text { paciencia y tolerancia. } \\
\checkmark \text { Comunicación de su enfermedad, } \\
\checkmark \text { tratamiento y cuidado hospitalario, } \\
\checkmark \text { según grado de dependencia. } \\
\checkmark \quad \text {-Trato respetuoso, amable y } \\
\checkmark \quad \text { cariñoso con ingenio y creatividad. } \\
\text { - Debilidades en la interacción enfermera-paciente: } \\
\checkmark \quad \text { Sobrecarga laboral. } \\
\checkmark \text { Carencias y deficiencias en el hospital produce estrés. } \\
\checkmark \text { No hay cultura de prevención. } \\
\checkmark \quad \text { Insatisfacción por complicación de enfermedad, no se cumple } \\
\text { - Fortalezas en la interacción enfermera-familiar: } \\
\checkmark \text { Conocer el nivel de instrucción e idiosincrasia del familiar. } \\
\text { - Debilidades en la interacción enfermera- familiar: } \\
\checkmark \text { Exigencia de informe médico por complicación de } \\
\checkmark \text { enfermedad. } \\
\checkmark \text { No respetan normas del hospital. }\end{array}$ \\
\hline $\begin{array}{l}\text { 4. Actitudes y valores } \\
\text { relacionados al cuidado } \\
\text { humanístico en el } \\
\text { entorno de los servicios } \\
\text { hospitalarios }\end{array}$ & $\begin{array}{l}\text { - Actitudes: } \\
\checkmark \text { Trabajo en equipo. } \\
\checkmark \text { Comunicación asertiva. } \\
\checkmark \text { Coordinación permanente. } \\
\checkmark \text { Liderazgo. } \\
\text { - Valores: } \\
\checkmark \text { Fortalecer el trato respetuoso. } \\
\checkmark \text { Humildad. } \\
\checkmark \text { Disciplina. } \\
\checkmark \text { Inteligencia emocional. } \\
\checkmark \text { Solidaridad. } \\
\checkmark \text { Responsabilidad. } \\
\checkmark \text { Honestidad. }\end{array}$ \\
\hline
\end{tabular}

Fuente: Base de datos 


\section{DISCUSIÓN.}

Las representaciones sociales (RS) permiten reconocer los modos y procesos de constitución del pensamiento por medio del cual las personas construyen $y$ son construidas por la realidad social, el significado ontológico que le dan a su ser intenta dominar el entorno (8). Para ejercer la enfermería es fundamental ser una persona con inspiración y llamado hacia la dedicación y la capacidad humana para decidir con libertad por el bienestar del paciente. Los relatos concuerdan con lo resaltado por Waldow (3) y Landam (10), la primera considera que, para cuidar a un paciente hospitalizado la enfermera debe poseer paciencia, atención, cariño, atender con voluntad, ser servicial y con gran profesionalismo; cualidades reconocidas como altruistas por la sociedad. La segunda afirma que la persona que se desempeña en los servicios de emergencia debe tener vocación, optimismo y tomar su labor como un desafío. Además, es importante saber escuchar al paciente, es dejarle hablar; es muy valioso encontrar un cuidador que sepa escuchar, sobre todo en momentos difíciles de la vida, ésta permite cierto grado de confianza y comprensión afirman Sánchez y Pinto (11).

Según Nightingale la enfermería es un llamado superior y honorable que radica en el amor y persistencia en el cuidado, es presencia genuina que permite el crecimiento personal y profesional, el yo personal tiene gran influencia en el yo profesional (12).

La razón existencial de la enfermería es el cuidado científico, el poder de una enfermera se incrementa con la disciplina (orden, limpieza y puntualidad) y el conocimiento teórico. Se considera además que el proceso de cuidado de enfermería es un instrumento científico que evidencia la ciencia de enfermería (13). Según Banch los conocimientos socialmente elaborados y compartidos bajos sus múltiples aspectos intentan dominar esencialmente nuestro entorno. Comprender y explicar los hechos e ideas que surgen en él, los descubrimientos de la ciencia y el devenir histórico es fundamental para la conducta de nuestra vida profesional (7).

En la actualidad enfermería se encuentra en el paradigma de la trasformación por cuanto las enfermeras reconocen la cuantiosa herencia científica; modelos y teorías; entre las más influyentes podemos citar a la teoría del entorno saludable, propuesta por Nightingale, teoría de la práctica humanística, propuesta por Paterson y Zderad, teoría de la evolución humana propuesta por Parse, modelo de sistemas propuesta por Neuman, seres humanos unitarios propuesta por Rogers, teoría del autocuidado propuesta por Orem, Filosofía y ciencia del cuidado propuesta por Watson, modelo de las catorce necesidades básicas propuesta por Henderson y la teoría transcultural propuesta por Leininger. Consideran conceptos de meta paradigmas, persona, salud, entorno y cuidado. En la actualidad, enfermería tiene como referente estas teorías y modelos y como elemento metodológico el proceso de atención de enfermería. Ambos elementos significan la epistemología de la enfermería (14), situación que genera la consideración de los amplios conocimientos que se deben poseer.

Valenzuela (15), considera que, para llevar a cabo el proceso de humanización es necesario un cambio de paradigma hacia un modelo holístico con habilidades relacionales, destacando la relación de ayuda y comunicación. Asimismo, Landam (10), considera al cuidado humanizado como integral e incluye a la familia del paciente considerando los principios bioéticos, limitaciones y facilidades dentro del ámbito laboral. Para Huercanos (16), los principales cuidados invisibles de enfermería, se dirigen al uso de estrategias comunicativas, verbales y no verbales, para el cuidado emocional de pacientes y familiares. 
El cuidado como esencia de enfermería lo sustentan Paterson y Zderad, consideran que la enfermería humanística implica respuesta humana y entiende científicamente al hombre, identificando la intensidad del dolor y sufrimiento, que induce al individuo a solicitar ayuda, acudiendo a centros hospitalarios (1).

Los discursos concuerdan con lo descrito por Guirao (17), quien sostiene que la noción de cuidado ocupa un lugar central $y$ fundamental en el discurso de enfermería y nos trae a reflexión un mito-fabula de Higinio del que Boff hace eco; en relación al cuidado; nuestros actos de cuidado influirán en el bienestar del hombre y le permitirán afrontar dignamente el proceso de vidamuerte. El cuidado como esencia de enfermería, debe estar sustentado científicamente considerando la experiencia existencial, relación transaccional y conciencia intelectual de las respuestas humanas, prestando atención a los valores humanos, valores sociales, tiempo y espacio.

La interacción favorable entre enfermera paciente y equipo de salud, ésta guiada por la práctica de la teoría humanística considerando la trilogía filosófica (existencialismo, humanismo y la fenomenología) (1,4) Las (RS) permiten entender la dinámica de las interacciones sociales y aclarar las determinantes de sus prácticas pues se generan mutuamente (8). La ciencia de enfermería está centrada en las relaciones transpersonales entre enfermera y paciente. La comunicación es una fortaleza fundamental en las interacciones personales, tiene una puerta de entrada que es la acogida, según Widenbach citada por Marriner (2), en ella es importante las expresiones verbales y no verbales, por tanto, los contenidos conativos (función lingüística imperativa) intentan dirigir la conducta del paciente y orientan sus prácticas de autocuidado.
Es muy importante identificar al paciente y clasificarlo según grados de dependencia: I, II, III y IV, para satisfacer sus necesidades, esto permite brindar un cuidado de calidad (18), defendiendo el cuidado cultural y profesional a fin de ser congruente, sensible y competente a las personas de diferentes culturas (2), con ingenio y creatividad según los informantes.

Los hospitales del Ministerio de Salud (MINSA) y del Seguro Social (ESSALUD) son mal vistos por la población, entre otras razones, por frecuente maltrato a los pacientes, prolongados tiempos de espera, falta de equipamiento y medicamentos; desaprobándolo al sistema de salud pública con 10,1 cuatro puntos por debajo del privado que alcanzó a 14 (19). Estas consideraciones se reafirman en el estudio pues existen carencias y deficiencias en el hospital estatal consideradas como debilidades. Frente a ello la/el enfermera/o trata de contribuir con el paciente, generando soluciones creativas, con aportes económicos voluntarios, préstamos de medicamentos, búsqueda de donaciones, para su recuperación oportuna y de este modo evitar costos innecesarios de estancia hospitalaria. Estos aportes realizados a la sociedad son pocas veces descritas y valoradas. Es evidente que aparte de las situaciones estresantes que vive el profesional de enfermería durante el cuidado a los pacientes de diversas patologías y grados de dependencia, se suman las carencias y deficiencias de equipos, materiales y medicinas, generándoles estrés sobredimensionado que influye en su desempeño profesional y familiar.

Casullo (20), considera que el trabajo en hospitales ha sido considerado intrínsecamente estresante de sobre adaptación, que conduce a un desgaste laboral. Chiang y col. (21), refieren que el $76 \%$ de enfermeros presentan estrés laboral y las más afectadas son mujeres adultas con roles de formación de pareja y crianza de hijos menores, de condición laboral 
contratada y que además tienen como factor predominante la sobrecarga de trabajo, se corrobora en el estudio.

Berlinguer (22), sostiene que los importantes cambios en el clima moral y político demuestran prioridades de los gobiernos, prefieren invertir más en la construcción de hospitales que en favor de la prevención de enfermedades. Debemos garantizar un mayor empoderamiento del individuo en el cumplimiento del ciclo vital y los deberes que le viene asignados. La enfermera (o) debe respetar los valores, usos hábitos, costumbres y creencias de la persona, familia y comunidad siempre que estos no pongan en riesgo su salud, considerando los principios bioéticos (23).

Cuanto mayor es el grado de instrucción del familiar, mejor es la capacidad de ayuda y colaboración con el paciente y profesional de salud, porque permite su involucramiento en el cuidado, esto es considerado como fortaleza durante el cuidado.

Una de las debilidades es la interferencia de la labor de enfermería por parte de los familiares, que solicitan información de la enfermedad de sus pacientes. La calidad de relación entre médico y paciente es importante, cuanto mejor sea en términos de respeto mutuo, compartidos mejor será el afrontamiento de la enfermedad por parte del paciente y sus familiares.

Moscovici, enfatiza la comprensión de la realidad social a partir de su construcción social e indica que en la interacción sujetoobjeto intervienen otros sujetos llamado Alter (A). En la interacción enfermera-paciente en el hospital además intervienen otros profesionales de la salud, que contribuyen a la recuperación del paciente (8). Likert, establece que el comportamiento de los subordinados depende directamente del comportamiento del administrativo y las condiciones organizacionales que influyen en sus actitudes (24). Watson, considera que el trabajo en equipo y el liderazgo son pilares del cuidado de enfermería (2).

Los discursos y las observaciones evidencian que en el equipo de salud hay escasa coordinación e interacción para el servicio al paciente y familiar. Es importante coordinar con otros profesionales y evitar servicios fragmentados, que generan malestar al paciente y familiar, ellos reaccionan mostrando actitudes negativas (enojos y gritos).

Campos (25), refiere que en todo espacio socio cultural las relaciones interpersonales son esenciales en la vida diaria y nos hace más humanos. Las normas de relación médicos-enfermeras son: respetar a las personas y tratar con cortesía, no invadir el área específica de trabajo, no delegar funciones propias, reconocer la legítima línea de mando y denunciar a quien se comporte descuidada o negligentemente. También las relaciones humanas de amistad, empatía, respeto mutuo y trabajo deben caracterizar la relación profesional según Guillen (26).

Los discursos concuerdan con lo planteado por Urquiaga (27), quien describe un cotidiano de vida de la enfermera e indica como una de las principales funciones a la administración priorizando la coordinación.

Según Waldow (3), la postura estética y ética del cuidado es la forma de vivir, de ser y de expresarse en enfermería. La humildad es el principio de grandes valores honorables, con ella se logra aprender y desarrollarse; para una gran personalidad. Por otro lado, consideran a la inteligencia emocional, que según Goleman (28), nos permite afrontar situaciones, pues las consideraciones de la época exigen dominio de sí mismo y compasión, es decir ponerles inteligencia a nuestras emociones. Al respecto Medina (29), refiere que en ninguna otra profesión se exige tanto rigor de orden moral como en las sanitarias. 
Ariza (30), considera que, para hablar de una atención humana en los escenarios del área de la salud es importante contemplar a los agentes que participan en ella (todos los profesionales de la salud) y las instituciones formadoras con valores y principios éticos, considerando que el respeto ocupa un lugar privilegiado, desarrollado en doble vía profesional-paciente, paciente-profesional y entre profesionales.

\section{CONCLUSIONES.}

Las representaciones sociales (RS) del cuidado de enfermería en los servicios hospitalarios, evidencian que existen debilidades en la interacción transpersonal, permitiendo la consideración de la conducta deshumanizante, por cuanto no se facilita las condiciones indispensables para un cuidado humanístico. Hay un ideal de pensamiento conforme a la ciencia y el pensamiento de la realidad en el medio social, que demuestra que hay separación entre valores científicos y valores sociales; la ciencia exige ciertas condiciones para el cuidado profesional y el entorno donde se practica no garantiza las condiciones, generando estrés en los profesionales de enfermería y la insatisfacción de los pacientes en los servicios hospitalarios.

\section{AGRADECIMIENTOS:}

Nuestra eterna gratitud al Dr. Miguel Oliveros Donohue asesor del estudio, Dra. Martha Vera Mendoza y Dra. Rocío Cornejo Valdivia por sus contribuciones.

A las/los enfermeras/os del Hospital Estatal de Cerro de Pasco Perú, fuente de conocimientos que permitió la investigación.

\section{CORRESPONDENCIA:}

Nelly Marleni Hinostroza Robles. Facultad de Ciencias de la Salud. EFPE, UNDAC, Cerro de Pasco, Perú, Filial Tarma.

Correo-e: nhinostrozar@undac.edu.pe

Cel. 945223183

\section{REFERENCIAS BIBLIOGRÁFICAS}

1. Paterson J, \& Zderad L. Enfermería Humanística. México: Limusa S.A; 1990.

2. Marriner A. Modelos y Teorías en Enfermería. $5^{\circ}$ ed. España: Ed. Elsevier S.A.; 2008: pp.147-50

3. Waldow V. Cuidado humano $\mathrm{O}$ resgate necessário. Porto Alegre: Zagra Luzzatto; 1998: p 51.

4. Watson J. El Cuidado. (Internet) (Citado el 7 de enero del 2015). Disponible en: http:// www. Teorías de enfermería.blogspot. com > je; 2012.

5. Zapata I. Situación del Cuidado de Enfermería en el Perú. IV Curso Internacional. Cuidado Humanizado. Compromiso Profesional de Enfermería con la Sociedad [Diapositiva] CEP Lima; 2011.

6. Espinoza L, et al. El cuidado humanizado y la percepción del paciente en el Hospital ESSALUD (Internet). Huacho, Perú; 2010. (Citado 05 de octubre del 2014). Disponible en www.uap.edu.pe/investigaciones/Esp/R evista 15 Esp.pdf

7. Banchs M. "Las representaciones sociales, sugerencias para una alternativa teórica y un rol posible para los psicólogos sociales en Latinoamérica" México: Universidad de Guadalajara; 1990.

8. Araya S. Las representaciones sociales. Ejes teóricos para su discusión. (Internet). Costa Rica: FLACSO (Citado 11 de octubre del 2014). Disponible en http:// www.or.cr>index.php.pdf; 2012.

9. Rodríguez G. Metodología de la Investigación Cualitativa. $2^{\circ}$ ed. Málaga España: Aljibe S.L.; 2008: p.223. 
10. Landam C, et al. "Cuidado Humanizado desde la perspectiva del enfermero de emergencia en un Hospital de Quillota Chile" Tesis Doctoral. (Internet). Universidad Alicante. Chile; 2014. (Citado 03 enero 2015). Disponible en: https://revistas.ucu.edu.uy/index.php/enf ermeriacuidadoshumanizados/article/.../ 564

11. Grupo Cuidado, Sánchez y Pinto. Dimensiones del cuidado. Colombia: Facultad de Enfermería; 1998: pp. 13, 191.

12. Leddy S, Pepper J. Bases Conceptuales de la Enfermería Profesional. Organización Panamericana de la Salud New York U.S.A: Edic. en Español; 1989: pp.63-7.

13. Bravo M. Guía Metodológica del Proceso de Atención de Enfermería. Lima, Peru: Grafica Jesús; 2005.

14. Luiris T. Los paradigmas como base del pensamiento actual en la profesión de enfermería. (Internet). Rev. Cubana de Educación Médica Superior 16 (4). (Citado 25 Agosto 2014). Disponible en https://www.imbiomed.com.mx/articulo.p $h p ? i d=15052$

15. Valenzuela M. ¿Es posible humanizar los cuidados de enfermería en los servicios de urgencia? Tesis Doctoral.( Internet). Chile; 2015. (Citado 15 de junio 2015) Disponible en: https://rua.ua.es/dspace/bitstream/1004 $\underline{5 / 50212 / 1 / \text { tesispdf }}$

16. Huercanos I. "El cuidado invisible, una dimensión de la profesión enfermera" Tesis Doctoral. Internet). España: Universidad Zaragoza; 2010. (Citado 05 de Mayo 2015) Disponible en: www.indexf.com/lascasas/documentos/lc0510.pd
17. Guirao J. La medida del cuidado. 5to Congreso Nacional AEC. Balneario de Archena (Internet). Murcia; 2004. (Citado 15 de Mayo 2015) Disponible en: www.uv.es/joguigo/materiales-dominioprofesional/medcuid.pdf

18. MINSA, Perú. Compendio de guías de intervenciones y procedimientos de Enfermería en Emergencias y Desastres. (Internet). Perú. (Citado 27 agosto 2015]. Disponible http:// www. minsa.gob.pe> org. Pdf. Normas; 2006.

19. El Comercio. PC>Móvil Peruanos desaprueban atención en hospitales del MINSA y ESSSALUD: 17 de marzo; 2014.

20. Casullo M. Evaluación del Bienestar Psicológico en Iberoamérica (Internet). Buenos aires: Ed. Paidós; 2002. (Citado 09 setiembre 2015). Disponible en: www.redalyc.org/pdf/801/80124028001. pdf

21. Chiang M, Salazar C, Nuñez A. Clima organizacional y satisfacción laboral en establecimiento de salud estatal: Hospital Tipo1. Chile Rev. Theoria 16(2): 2007: pp.61-76.

22. Berlinguer G. Ética de la Salud. Buenos Aires: Lugar Editorial; 2009: pp. 53-55.

23. Colegio de Enfermeros del Perú CEP. Código de Ética y Deontología. Lima, Perú pp. 16, 31; 2005.

24. Brunet L. El clima de trabajo en las organizaciones. Mexico: Editorial Trillas; 1989: pp. 15-17.

25. Campos A. Importancia de las relaciones interpersonales en los Cuidados de Enfermería. (Internet). México: U. del Valle; 2006. (Citado 20 setiembre 1015). Disponible en: www.scielo.cl/scielo.php?pid=S0717$\underline{532015000100009 \& \text { script=sci arttex }}$ 
26. Guillén M. Habilidades de comunicación en relación médico-enfermera. (Citado 12 octubre 2015). Recuperado de http://scielo.sld.cu/scielo.php?script=sci arttext\&pid=S0864-

03192011000300006; La Habana Cuba, julio-septiembre del 2011

27. Urquiaga $\mathbf{M}$. "Vivencias de los profesionales de enfermería en la gestión del cuidado a personas hospitalizadas. Hospital San Ignacio de Casma" Tesis Doctoral. (Internet). ULADECH, Perú; 2015: p.52. (Citado 20 enero 2016). Disponible en: http://repositorio.uladech.edu.pe/handle/ $\underline{123456789 / 74}$

28. Goleman D. La inteligencia emocional. Ed. Verlap S.A. Buenos Aires Argentina; 1996: p.16.
29. Medina C. Ética y Legislación. MadridValencia: DAE; 2002: p.35

30. Ariza C. "Soluciones de Humanización en Salud en la práctica diaria" Tesis Doctoral. (Internet). Universidad Javeriana. Colombia; 2012. (Citado el 20 enero 2016). Disponible en www.scielo.org.mx/scielo.php?script=sci arttext\&pid=S1665

Recibido: 23/08/2020

Aprobado para publicación: 24/11/2020 